\title{
COMPARISON AND ANALYSIS OF ACCURACY OF ELEVATION EXTRACTION BASED ON THE ZY-3 01 AND 02 SATELLITES STEREOSCOPIC IMAGES
}

\author{
Liping Zhao ${ }^{1, *}$, Xingke Fu ${ }^{1}$, Xianhui Dou ${ }^{1}$, Hui Liu ${ }^{2}$, Zhi Fang ${ }^{2}$ \\ ${ }^{1}$ Satellite Surveying and Mapping Application Center, National Administration of Surveying, Mapping and Geoinformation, Beijing \\ 100048, China - (zhaolp, fxk, douxh)@sasmac.cn \\ ${ }^{2}$ Sichuan Second Institute of Surveying and Mapping Geographic Information Engineering, Chengdu 610100,China - \\ 6508490@qq.com, fangzhiblue@163.com
}

Commission III, ICWG IV/III

KEY WORDS: ZY-3, Stereoscopic, Satellite, GSD, DEM, differential DEM, Accuracy

\begin{abstract}
:
The ZY-3 is the civil high-resolution optical stereoscopic mapping satellite independently developed by China. The ZY-3 constellation of the twin satellites operates in a sun-synchronous, near-polar, circular $505 \mathrm{~km}$ orbit, with a descending location time of 10:30 AM and a 29-day revisiting period. The panchromatic triplet sensors, pointing forward, nadir, and backward with an angle of $22^{\circ}$, have excellent base-to-height ratio, which is beneficial to the extraction of DEM. In order to extract more detailed and highprecision DEM, the ZY-3 (02) satellite has been upgraded based on the ZY-3 (01), and the GSD of the stereo camera has been optimized from 3.5 to 2.5 meters.
\end{abstract}

In the paper case studies using the ZY-3 01 and the 02 satellite data for block adjustment and DEM extraction have been carried out in Liaoning Province of China. The results show that the planimetric and altimetric accuracy can reach 3 meters, which meet the mapping requirements of 1:50,000 national topographic map and the design performance of the satellites. The normalized elevation accuracy index (NEAI) is adopted to evaluate the twin satellite stereoscopic performance, and the NEAIs of the twin ZY-3 satellites are good and the index of the ZY-3(02) is slightly better. The comparison of the overlapping DEMs from the twin ZY-3 satellites and SRTM is analysed. The bias and the standard deviation of all the DEMs are better than 5 meters. In addition, in the process of accuracy comparison, some gross errors of the DEM can be identified, and some elevation changes of the DEM can also be found. The differential DEM becomes a new tool and application.

\section{INTRODUCTION}

The ZY-3 is the civil high-resolution optical stereoscopic mapping satellite independently developed by China. It is mainly used for 1: 50,000 scale topographic mapping. One of the distinguishing features of the $\mathrm{ZY}-3$ is that the panchromatic triplet stereo camera can work continuously for long periods of time to obtain thousands of kilometres of continuous strip data. The working mode is suitable for wide-range stereoscopic mapping, in particular global DEM extraction. In addition, the triplet data acquired by the panchromatic stereo camera, in combination with the multi-spectral cameras in 4 bands (blue, green, red and near infrared bands), can be widely used in the fields such as land resources, agriculture, forestry, environment and disaster reduction.

The ZY-3 (01) satellite was launched on January 9, 2012, and the ZY-3 (02) satellite was successfully launched on May 30, 2016. After the completion of on-orbit testing, the two satellites operate at a phase angle of $180^{\circ}$, forming a stereoscopic mapping satellite constellation. The ZY-3 constellation of satellites operates in a sun-synchronous, near-polar, circular $505 \mathrm{~km}$ orbit, with a descending location time of 10:30 AM and a 29-day revisiting period. The panchromatic triplet sensors, pointing forward, nadir, and backward with an angle of $22^{\circ}$, have excellent base-to-height ratio, which is beneficial to the extraction of digital elevation model (DEM). In order to extract more detailed and high-precision DEM, the ZY-3 (02) satellite has been upgraded based on the ZY-3 (01), and the GSD of the stereo camera has been optimized from 3.5 to 2.5 meters.

The ZY-3 satellite has the advantages of high geolocation accuracy and structural stability. Based on the high precision gyros and star sensors on the satellite, the arc seconds level attitude measurement accuracy is obtained. The centimetres level orbit determination accuracy is achieved with a dualfrequency GPS receiver (Cao et al. 2012).

The DEMs are is the basic data for many earth science studies, and are also an important support for many applications, such as land planning, ecological protection and restoration of farmland, lakes, forests, grassland, etc.

The ZY-3 performance for the bundle adjustment, DEM extraction and orthophoto generation have evaluated and compared against SPOT-5 HRS and Cartosat-1 DEM (d'Angelo 2013, Jacobsen2016, Jacobsen 2017). The quality of WorldDEM, AW3D30, SRTM DEM and ASTER GDEM2 have been analysed in many literatures (Bayburt et al. 2017, Tadono 2012).

* Corresponding author 
In this paper the accuracy of the bundle adjustment and DEM generation with the overlapping ZY-3 data is studied firstly, and the normalized elevation accuracy index (NEAI) is adopted to evaluate the twin satellite stereoscopic performance. Then the accuracy of the DEMs from the twin ZY-3 satellites and SRTM is analyzed and compared using the differential DEM method.

\section{DATASETS}

The triplet stereo image data of twin ZY-3 satellites with no cloud cover and clear features in Liaoning Province of China is used to analysis the precision. The ZY-3 01 and the 02 satellite data are obtained on the 17th May 2016 and 27th August 2016, and the GSD of the stereo image pair is $3.5 \mathrm{~m}$ and $2.5 \mathrm{~m}$ respectively.

This produces a base - height ratio of 0.88 between backward and forward image, which is suitable for cartographical mapping and DEM extraction. Table 1 is a list of GSD parameters of the image data. The cover range and thumb image of the overlapping $\mathrm{ZY}-3(01)$ and $\mathrm{ZY}-3(02)$ are shown in Figure 1 and Figure2.

133 well-distributed GCPs ranging from $11 \mathrm{~m}$ to $115 \mathrm{~m}$ in height over the entire area are used in the experiment. The GCPs are measured via RTK GPS with centimeter-level accuracy in the horizontal and vertical coordinates. Their image coordinates are measured via digital photogrammetric workstation with accuracy reaching sub-pixel level. Most of the GCPs are grouped in sites of twin points, the accuracy and reliability are increased because of the redundancy of the groups of GCPs.

\begin{tabular}{|c|c|c|c|}
\hline \multicolumn{2}{|c|}{ Satellite } & ZY-3(01) & ZY-3(02) \\
\hline \multicolumn{2}{|c|}{ Acquisition Date } & $2016-05-17$ & $2016-08-27$ \\
\hline \multirow{2}{*}{$\begin{array}{c}\text { Mean } \\
\text { GSD }\end{array}$} & Forward & $3.55 \mathrm{~m}$ & $2.51 \mathrm{~m}$ \\
\cline { 2 - 4 } & Backward & $3.56 \mathrm{~m}$ & $2.50 \mathrm{~m}$ \\
\cline { 2 - 4 } & Nadir & $2.10 \mathrm{~m}$ & $2.12 \mathrm{~m}$ \\
\hline
\end{tabular}

Table 1. Analyzed ZY-3 Image GSD in the experiment

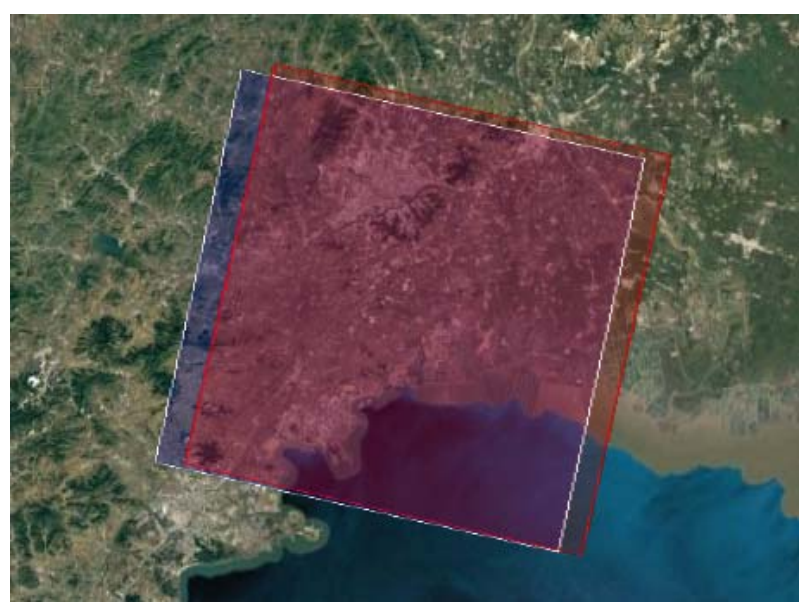

Figure 1. The cover range of the overlapping ZY-3(01) and ZY$3(02)$ in the experiment

Red:ZY-3(01), Blue:ZY-3(02)

\section{EXPERIMENTS}

In this evaluation the vendor supplied RPCs are used in bundle adjustment and DEM extraction, and affine transformation compensation models in image space with ground control points are used to improve the accuracy.
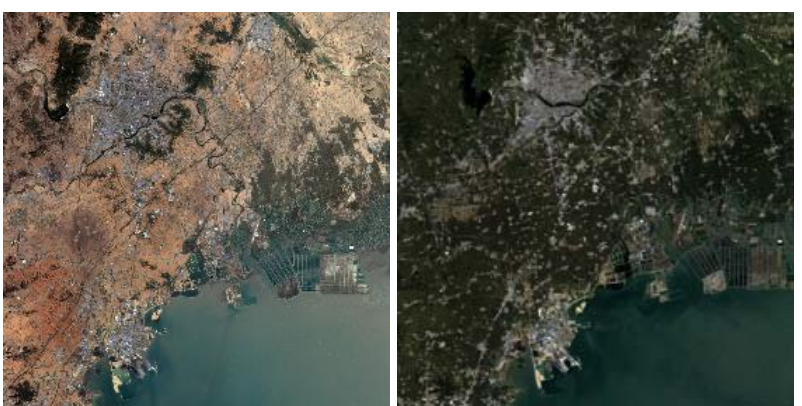

Figure 2. Thumb image of the ZY-3(01) and ZY-3(02) in the experiment (Left: ZY-3(01), right: ZY-3(02))

\subsection{Direct georeference}

Generally, the direct georeference accuracy is the base for selecting which geometric correction model, it is possible to compute the a-priori sensor accuracy by check points. Table 2 shows the accuracy of the ZY-3 triplet stereo images using direct georeferencing model based on rational function model with 133 independent check points.

From the standard deviation data analysis, it was found that the internal consistency of the ZY-3 data is very good, less than one GSD, and the accuracy of specification requirements can be achieved by using the bias model correction. However, in order to avoid the influence of the potential drift in time of the image position and the other tiny affine deformations existing, an affine correction model in image space is used in this study (Grodecki 2001, Jacobsen 2007).

\begin{tabular}{|c|c|r|r|r|}
\hline Satellite & & $\mathrm{N}(\mathrm{m})$ & \multicolumn{1}{c|}{$\mathrm{E}(\mathrm{m})$} & \multicolumn{1}{c|}{$\mathrm{H}(\mathrm{m})$} \\
\hline \multirow{2}{*}{ ZY-3(01) } & Bias & -3.216 & -19.112 & -12.029 \\
\cline { 2 - 5 } & Std.Dev & 1.625 & 1.386 & 2.634 \\
\hline \multirow{2}{*}{ ZY-3(02) } & Bias & 19.753 & 13.694 & -11.725 \\
\cline { 2 - 5 } & Std.Dev & 1.204 & 1.145 & 1.409 \\
\hline \multirow{2}{*}{ ZY-3(01+02) } & Bias & 10.167 & -0.599 & -20.071 \\
\cline { 2 - 5 } & Std.Dev & 1.130 & 1.097 & 1.096 \\
\hline
\end{tabular}

Table 2. The bias and the standard deviation of the ZY-3 triplet stereo images using direct georeferencing model with 133 independent check points

\subsection{Bundle adjustment}

Firstly, in order to evaluate trustworthily the planimetric and altimetric accuracy of ZY-3 stereoscopic images and verify the accuracy of the control points, all 133 points as control points are used to calculate the root mean square error (RSME) and standard deviation (Std.Dev.). The frequency distribution of the error of the points basically conforms to the normal distribution.

Then the eight well-distributed points composed of the four twin-point groups covered four corners in each stereo-pairs are used as ground control points and the remaining are used as independent check points. The bundle block adjustment based on affine model is then performed. The RSM error and the standard deviation of GCPs/ICPs after the adjustment are presented in Table 3, and the planimetric and altimetric accuracy are better than one GSD Obviously.

In this paper the normalized elevation accuracy index (NEAI) (formula 1) (Jacobsen, 2016) is used to analyze elevation accuracy avoiding the different GSD of the ZY-3 twin satellites. 
All the NEAI are around 0.5 and the NEAI of $Z Y-3(02)$ is better than the ZY-3(01) (Table 4).

$$
\text { NEAI = Accuracy } /(\text { GSD } \times \text { Height } / \text { Base })
$$

\begin{tabular}{|c|c|c|c|c|c|c|c|c|}
\hline \multirow{2}{*}{ Sat. } & \multirow{2}{*}{$\begin{array}{l}\text { GCPs/ } \\
\text { ICPs }\end{array}$} & & \multicolumn{2}{|c|}{$\mathrm{N}(\mathrm{m})$} & \multicolumn{2}{|c|}{$\mathrm{E}(\mathrm{m})$} & \multicolumn{2}{|c|}{$\mathrm{H}(\mathrm{m})$} \\
\hline & & & GCP & ICP & GCP & ICP & GCP & ICP \\
\hline \multirow{4}{*}{$\begin{array}{c}\text { ZY-3 } \\
(01)\end{array}$} & \multirow{2}{*}{$\begin{array}{c}133 / \\
0\end{array}$} & RSME & 1.33 & & 1.37 & & 1.98 & - \\
\hline & & StdDev & 1.33 & - & 1.37 & - & 1.98 & - \\
\hline & \multirow{2}{*}{$\begin{array}{c}8 / \\
125\end{array}$} & & 0.97 & 1.36 & 0.64 & 1.48 & 2.31 & 2.17 \\
\hline & & StdDev & 0.97 & 1.36 & 0.64 & 1.47 & 2.31 & 2.17 \\
\hline \multirow{4}{*}{$\begin{array}{c}Z Y-3 \\
(02)\end{array}$} & \multirow{2}{*}{$\begin{array}{c}133 \\
/ 0\end{array}$} & RSME & 1.15 & - & 1.12 & - & 1.23 & - \\
\hline & & StdDev & 1.15 & 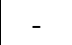 & 1.12 & - & 1.23 & - \\
\hline & \multirow{2}{*}{$\begin{array}{c}8 / \\
125\end{array}$} & RSME & 0.59 & 1.22 & 1.04 & 1.15 & 0.89 & 1.28 \\
\hline & & StdDev & 0.59 & 1.21 & 1.04 & 1.14 & 0.89 & 1.27 \\
\hline
\end{tabular}

Table 3. The RSM error and the standard deviation of GCPs/ICPs in the ZY-3 triplet stereo images with affine compensation model adjustment

\begin{tabular}{|c|c|c|c|c|c|}
\hline Satellite & GCPs/ICPs & $\begin{array}{c}\text { RMSE } \\
\mathrm{H}(\mathrm{m})\end{array}$ & $\begin{array}{c}\text { Mean } \\
\text { GSD }\end{array}$ & $\begin{array}{c}\text { GSD } \times \\
\text { H/B }\end{array}$ & NEAI \\
\hline \multirow{2}{*}{ ZY-3(01) } & $133 / 0$ & $\mathbf{1 . 9 8}$ & 3.5 & 3.99 & $\mathbf{0 . 5 0}$ \\
\cline { 2 - 6 } & $125 / 8$ & $\mathbf{2 . 1 7}$ & 3.5 & 3.99 & $\mathbf{0 . 5 4}$ \\
\hline \multirow{2}{*}{ ZY-3(02) } & $133 / 0$ & $\mathbf{1 . 2 3}$ & 2.5 & 2.85 & $\mathbf{0 . 4 3}$ \\
\cline { 2 - 6 } & $125 / 8$ & $\mathbf{1 . 2 8}$ & 2.5 & 2.85 & $\mathbf{0 . 4 5}$ \\
\hline
\end{tabular}

Table 4 . The normalized elevation accuracy index of the ZY-3 triplet stereo images

\subsection{DEM generation}

After bundle adjustment, the DEM generation is realized from backward-forward image pairs of the ZY-3 twin satellites according to the Chinese 1:50000 scale DEM national specification and the space grid is $10 \mathrm{~m}$ for Digital Surface Model (DSM) and 25m for Digital Terrain Model (DTM)

In the DEM production process, an automatic filtering and manual editing are applied in order to remove gross errors in some difficult areas such as reservoirs, lakes, rivers, etc.

The DSM from the ZY-3(02) and DTM from the ZY-3(01) are shown in Figure 3. The building surface is obvious in the DSM (Fig. 3, left). For objective comparison, all check points are located on partially open and flat terra surfaces. The elevation RSME of check points is listed in Table 5, which meet the national mapping requirements of 1:50,000 scale. In addition, the normalized elevation accuracy index is calculated (Table 5) and the NEAI of ZY-3 (02) seems to be better.
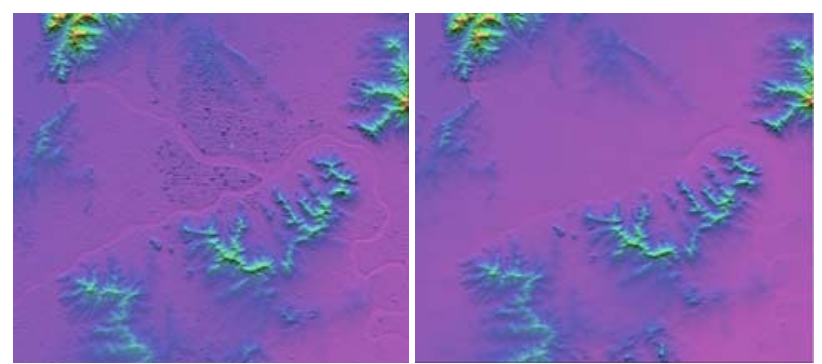

Figure 3. The DSM from the ZY-3(02) (left), DTM from the $\mathrm{ZY}-3(01)$ (right)

\begin{tabular}{|c|c|c|c|c|c|c|c|}
\hline Sat & Product & CPs & Grid & Bias & StdDev & RMSE & NEAI \\
\hline $\begin{array}{c}\text { ZY-3 } \\
(01)\end{array}$ & DSM & 22 & 10 & -0.662 & 2.210 & 2.308 & $\mathbf{0 . 5 7 8}$ \\
\cline { 2 - 8 } & DTM & 22 & 25 & -1.728 & 2.006 & 2.647 & $\mathbf{0 . 6 6 3}$ \\
\hline $\begin{array}{c}\text { ZY-3 } \\
(02)\end{array}$ & DSM & 24 & 10 & 0.203 & 1.473 & 1.487 & $\mathbf{0 . 5 2 2}$ \\
\cline { 2 - 8 } & DTM & 24 & 25 & -0.967 & 1.401 & 1.702 & $\mathbf{0 . 5 9 7}$ \\
\hline
\end{tabular}

Table 5. The DSM and DTM accuracy of the ZY-3 twin satellites (unit: meter)

\section{COMPARISON AND ANALYSIS}

The bundle adjustment accuracy and DEM extraction accuracy from the twin satellites stereoscopic image data is satisfactory. In order to further analyze the accuracy of the ZY-3 satellites, the horizontal and vertical accuracy of the twin DEM products are evaluated and compared. In addition, accuracy comparisons are performed with the SRTM DEM data.

\subsection{DSM Comparison of the twin satellites}

Difference between the digital surface models with the grid spacing of $10 \mathrm{~m}$ from the twin satellites has been computed. Horizontal registration error and height discrepancy are analyzed, the statistics are showed in Table 6 and the corresponding frequency distribution chart is described in Figure 4. The horizontal bias (table 6) is almost negligible since the twin DSMs production based on the same GCPs.

\begin{tabular}{|c|c|c|c|}
\hline & $\mathrm{E}$ & $\mathrm{N}$ & $\mathrm{H}$ \\
\hline Bias & 0.250 & -0.591 & -1.546 \\
\hline Std.Dev. & 4.417 & 4.469 & 3.559 \\
\hline
\end{tabular}

Table 6 . The bias and the standard deviation of the differential DSM between the twin ZY-3 DSMs (unit: meter)

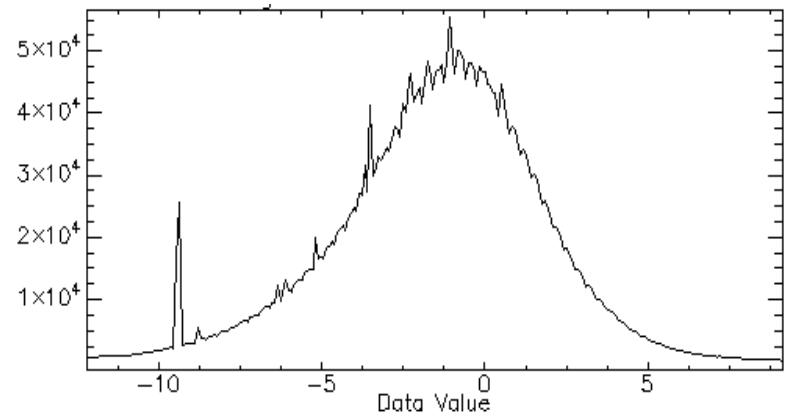

Figure 4. Frequency distribution of the differential DSM between the twin ZY-3 DSMs

The color mapping of the differential DSM (D-DSM) by the standard deviation can be used to identify the big height discrepancies (figure 5). The size of discrepancies is indicated in the order of red, yellow, blue, and green, respectively, where the red region indicates greater than 3 times standard deviation.

The bias and standard deviation of height in the tables 5 and 6 is analyzed, and the function can be described by Formula 2 .

$$
\sigma_{1}^{2}+\sigma_{2}^{2}<<\sigma_{d}^{2}
$$

where $\sigma_{1}, \sigma_{2}$ and $\sigma_{d}$ are the standard deviation of accuracy of the ZY-3(01) DSM, ZY-3(02) DSM and differential DSM respectively. 


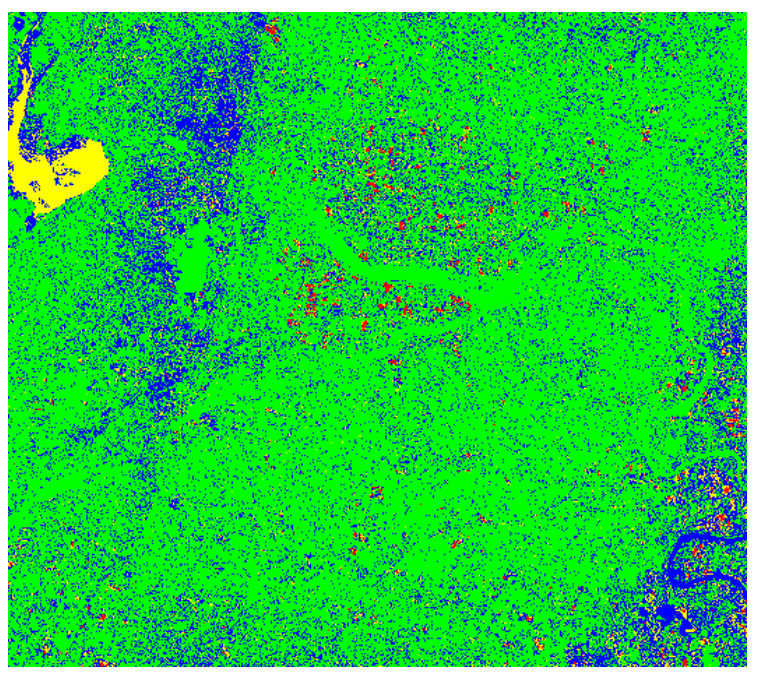

Figure 5. Color mapping of the differential DSM between the twin ZY-3 DSMs according to the standard deviation

Since the accuracy of the DSM is calculated from a small number of well-defined checkpoints distributed in relatively local flat areas, the validation points cannot fully represent the DSM accuracy. Under flat terrain conditions, it is advantageous to improve image matching accuracy and reduce the quantization errors for DSM interpolation, and the slight offset of the DSM in the plane has little effect on the detection of the elevation accuracy. On the other hand, and the 25-meter grid will also cause a slight decrease in the height accuracy with respect to the 2.5-meter GSD size. Therefore, the actual DSM accuracy is lower than the value in the table 5.

In addition, from the analysis of formula 2, there may be gross errors or changes in terra surface elevation in the two DSMs. Since there is a difference of about 100 days between the data in the two periods, the probability of a significant change in the artificial target within a short period of time is less likely. It is assumed that the possibility of changes due to natural factors is greater. From the analysis of the acquisition time of the two periods of data, it is just in the period of alternating spring and summer. Combined with the geographic location of the experimental area, the precipitation and vegetation growth may change significantly.

An abnormal peak around $-9.6 \mathrm{~m}$ of the height discrepancy distribution can be seen in figure 4 , and the peak data happens to be mainly distributed in the yellow region of figure 5 and the corresponding ZY-3 01 and 02 image data is shown in figure 6.

Through comparison of images, it is found that the storage area of the reservoir varies greatly and is very obvious, resulting in a change in the water surface elevation and showing an abnormal peak in the differential DSM. Moreover changes in the water surface of the river are also found in the image shown in Figure 7, which can also be clearly shown in Figure 6.

In this paper the height discrepancy ranges from $-20 \mathrm{~m}$ to $-70 \mathrm{~m}$ in the red region is analyzed, as shown in Figure 7 . The gross errors in the DSM generated from the ZY-3(01) stereo images are mainly due to a mismatch in the areas of high-rise buildings and their shadows with low resolution.

In addition, there may be other types of surface changes and gross errors in the DSMs, which require further investigation.

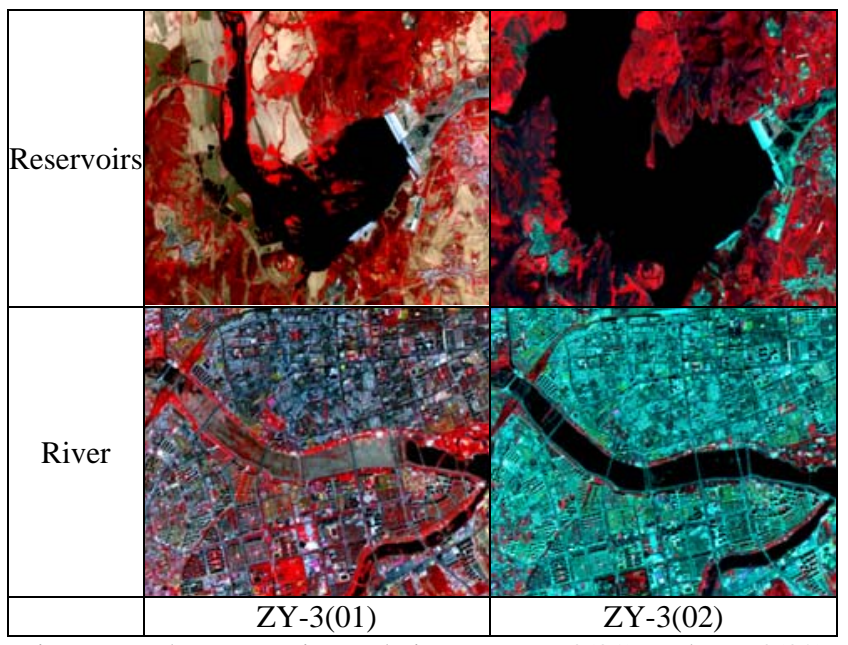

Figure 6. The reservoirs and river on $\mathrm{ZY}-3(01)$ and $\mathrm{ZY}-3(02)$ false color images

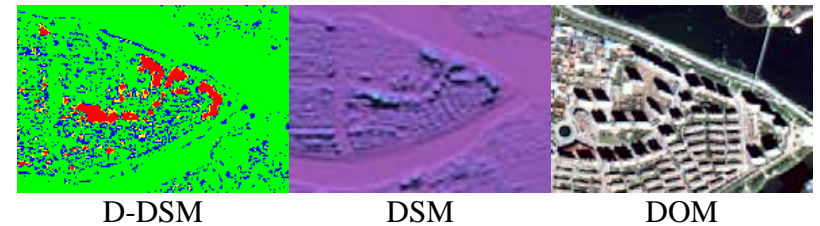

Figure 7. The high-rise building on D-DSM, DSM and DOM

\subsection{DEM Comparison with SRTM}

In order to further evaluate the accuracy of DEM data, a comparative analysis is performed with SRTM DEM data. First, the two DEMs with different height systems are converted from the orthometric height above the corresponding geoid to the ellipsoid height above the WGS84 ellipsoid (Zhao et al. 2018). In addition, because the points are not registered between two DEMs, the SRTM DEM data is reprojected and interpolated with the grid spacing of $25 \mathrm{~m}$ according to the ZY-3(02) DEM, and then the difference between DEMs is calculated.

The planimetric and altimetric difference between the two DEMs are showed in Table 7 and the corresponding frequency distribution chart and the color mapping of the differential DEM are described in Figure 8 and Figure 9. It is amazing that the horizontal bias and height discrepancy of the overlapping DEMs based on different Optical and Synthetic Aperture Radar data are very small (table 7).

Obviously, some big discrepancies in the red region are visible in the color mapping of the differential DEM (figure 9). In order to verify the height difference, we first compared the corresponding DEM and image data of the ZY-3, and confirmed that there was no gross error in the DEM extraction. In terms of SRTM DEM, the contemporaneous image data is collected from the Google Earth historical image database according to the mission time of SRTM on the Space Shuttle in February 2000.

\begin{tabular}{|c|c|c|c|}
\hline & $\mathrm{E}$ & $\mathrm{N}$ & $\mathrm{H}$ \\
\hline Bias & -0.794 & -1.226 & 1.526 \\
\hline Std.Dev. & 6.177 & 7.338 & 3.225 \\
\hline
\end{tabular}

Table 7. The bias and the standard deviation of the differential DEM between the ZY-3 DEM and SRTM DEM (unit: meter) 


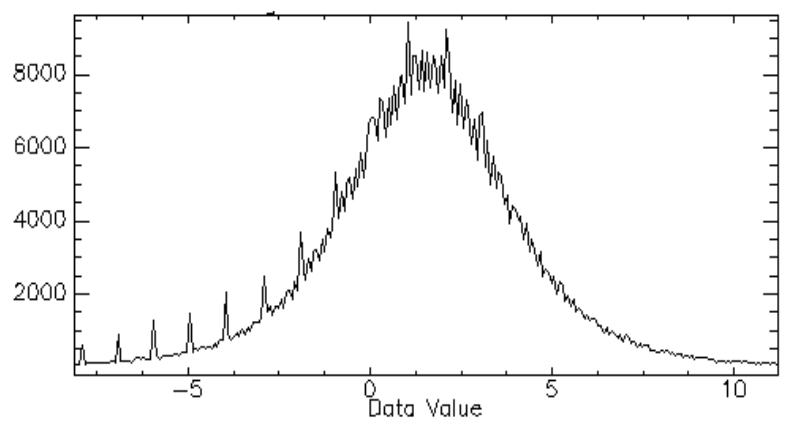

Figure 8. Frequency distribution of the differential DEM between the ZY-3 DEM and SRTM DEM

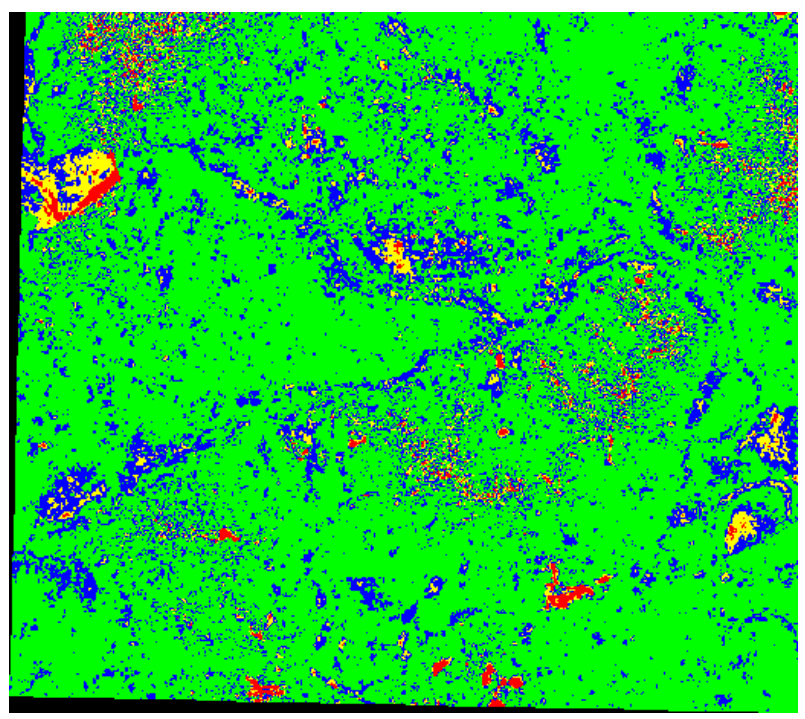

Figure 9. Color mapping of the differential DEM between the ZY-3 DEM and SRTM DEM according to the standard deviation

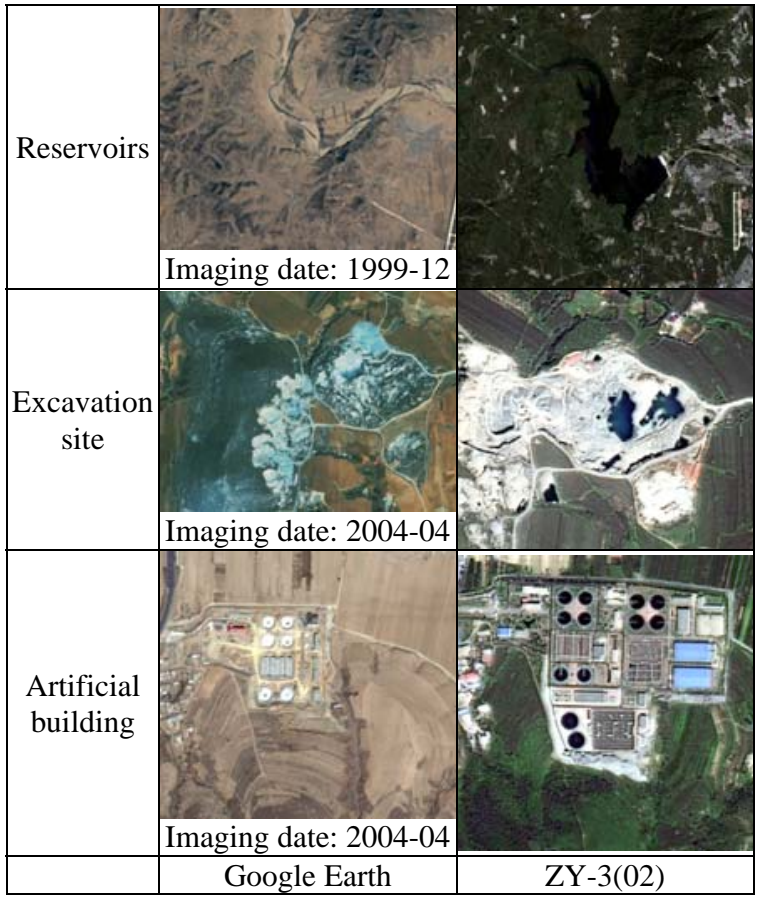

Figure 10. The reservoirs, excavation site and artificial building on the Google Earth and ZY-3(02) images

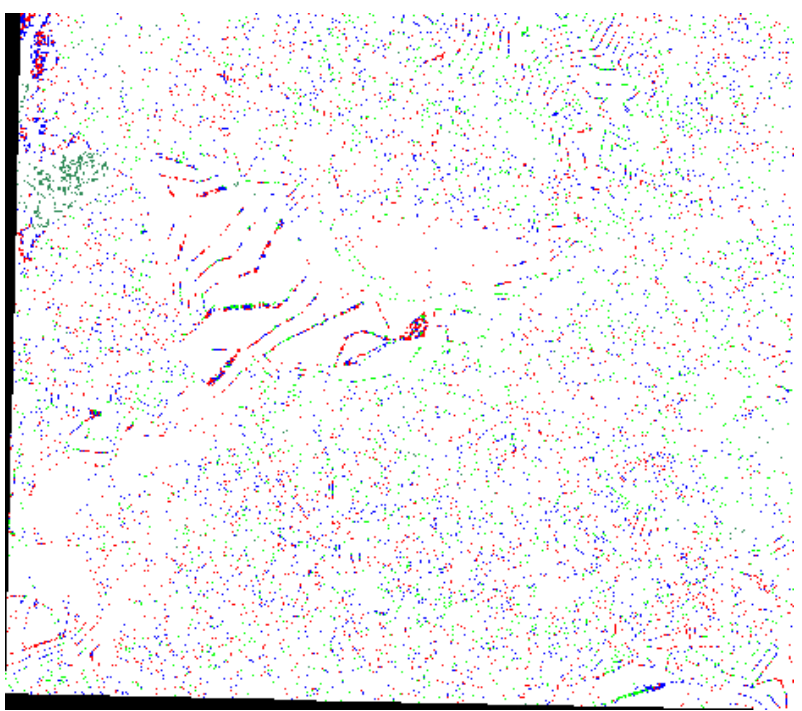

Figure 11. The color coded map of peaks in the frequency distribution of the differential DEM

As shown in Figure 10, the left image is Google Earth historical data, and the right is ZY-3 (02) data. From top to bottom, reservoirs, excavation sites, and artificial buildings are respectively listed.

After comparative analysis, they can be found on the Google Earth images that the reservoir has not yet been built, and the scope of the excavation sites and artificial buildings is also small. On the latest ZY-3 imagery, changes in the ground cover and ground elevations have been found in these areas, among which the variation range of the reservoir elevation is from $-3 m$ to $-20 \mathrm{~m}$, and the average height change of the excavation site and the artificial building is $38 \mathrm{~m}$ and $18 \mathrm{~m}$, respectively.

Several slight abnormal peaks of the height discrepancy distribution chart can be seen in figure 8 , but the cause is difficult to find after preliminary analysis of the color coded map of the peaks (figure 11).

\section{CONCLUSION}

The bundle adjustment and DEM generation with the overlapping ZY-3 01 and 02 triplet stereo image data in Liaoning Province of China in this paper is tested and the results show that the planimetric and altimetric accuracy can reach 3 meters, which meet the mapping requirements of 1:50,000 national topographic map and the design performance of the satellites.

In order to avoid the influence of different GSDs and baseheight ratio when evaluating the accuracy, the normalized elevation accuracy index (NEAI) is adopted in this paper. The NEAIs of the twin ZY-3 satellites are good and the index of the $\mathrm{ZY}-3(02)$ is slightly better.

The comparison of the overlapping DEMs from the twin ZY-3 satellites and SRTM is analyzed. The bias and the standard deviation of all the DEMs are better than 5 meters.

In addition, in the process of accuracy comparison, some gross errors of the DEM can be identified, and some elevation changes of the DEM can also be found. The differential DEM becomes a new tool and application. 


\section{REFERENCES}

Bayburt, S., Kurtak, A. B., Büyüksalih, G., and Jacobsen, K., 2017. Geometric accuracy analysis of WORLDDEM in relation to AW3D30, SRTM and ASTER GDEM2, Int. Arch. Photogramm. Remote Sens. Spatial Inf. Sci., XLII-1/W1, 211217, 2017.

Cao Haiyi, Liu Xigang, Li Shaohui and Zhang Xinwei, 2012. ZY-3 Satellite Remote Sensing Technology, Spacecraft Recovery \& Remote Sensing, 33, pp. 7-16

d'Angelo, P., 2013. Evalution of ZY-3 for DSM and ortho image generation, Int. Arch. Photogramm. Remote Sens. Spatial Inf. Sci., XL-1/W1, 57-61, 2013.

Grodecki, J., 2001. IKONOS Stereo Feature Extraction - RPC Approach, ASPRS annual conference St. Louis 2001

Jacobsen, K., 2007. Orientation of high resolution optical space images, ASPRS annual conference, Tampa 2007

Jacobsen, K., 2016. Analysis and correction of systematic height model errors, Int. Arch. Photogramm. Remote Sens. Spatial Inf. Sci., XLI-B1, 333-339, 2016.

Jacobsen, K., 2017. Problems and limitations of satellite image orientation for determination of height models, Int. Arch. Photogramm. Remote Sens. Spatial Inf. Sci., XLII-1/W1, 257264, 2017.

Tadono, T., Takaku, J., and Shimada, M., 2012. Validation study on ALOS PRISM DSM mosaic and ASTER GDEM 2, ISPRS Ann. Photogramm. Remote Sens. Spatial Inf. Sci., I-4, 193-198, 2012.

Zhao Liping, Fu Xingke, Zhu Guangbin, Zhang Jianhua, Han Chaobin and Cheng Lu, 2018. Discussion on height systems in stereoscopic mapping using the ZY-3 satellite images, ISPRS TC III Symposium 2018, Beijing, China. (Accepted) 